\title{
Surgical presentation of ischaemic hepatitis
}

\author{
L Powell, S Tesfaye, R Ackroyd, D S Sanders
}

Postgrad Med J 2003;79:350-351

Intestinal ischaemia typically presents with signs suggestive of an acute surgical abdomen. A raised serum lactate may substantiate the diagnosis. The first case of ischaemic hepatitis mimicking intestinal ischaemia is reported. The recognition of markedly deranged transaminases allows the clinician to differentiate the two diagnoses and potentially avoid the risks incurred with laparotomy.

schaemic hepatitis is a rare condition accounting for $0.16 \%$ $0.50 \%$ of inpatient admissions. ${ }^{1}$ Typically the patient has no abdominal findings and the diagnosis is based on biochemical parameters. ${ }^{2}$ There is a rapid and marked increase in liver enzymes, more than eight times the upper limit of normal, with normalisation within seven to 10 days and exclusion of viral causes or hepatotoxic agents as the cause. ${ }^{2}$ In addition, the serum lactate may also be raised. Ischaemic hepatitis can occur after periods of haemodynamic instability or hypoxia such as haemorrhage, sepsis, pulmonary embolus, cardiac failure, acute myocardial infarction, and other causes of respiratory distress. ${ }^{1-5}$

Ischaemic hepatitis is also known as "shock liver", but patients can develop this without episodes of hypotension. This is because the portal blood supply (providing $70 \%$ of the liver's blood supply) rather than systemic blood pressure is the critical determinant of hepatic perfusion. ${ }^{6}$ In addition, a reduced cardiac output leads to hepatic hypoxia and after activation of the renin-angiotensin system there is constriction of the splanchnic blood vessels, which maintains blood pressure, but reduces portal blood flow. ${ }^{6}$ The histological appearances at postmortem examination are of centrilobular necrosis with varying degrees of sinusoidal congestion..$^{1-5}$

We describe the first case of ischaemic hepatitis mimicking intestinal ischaemia.

\section{CASE REPORT}

A 77 year old male ex-smoker was admitted with a three day history of coffee ground vomiting and shortness of breath. His past medical history included hypertension, renal impairment, a right hemicolectomy for colonic cancer, and a laryngectomy for squamous cell carcinoma of the vocal cords.

On examination he was cyanosed, tachycardic, tachypnoeic, hypotensive (blood pressure 90/60 $\mathrm{mm} \mathrm{Hg}$ ), and clinically anaemic. His cardiovascular, respiratory, and abdominal examinations were unremarkable. On rectal examination he was found to have malaena. A diagnosis of upper gastrointestinal bleeding (of unknown aetiology) was made. His haemoglobin was $60 \mathrm{~g} / \mathrm{l}$ and a transfusion was instituted. His condition progressively deteriorated and six hours after admission he developed abdominal signs of generalised tenderness, guarding in both flanks, and absent bowel sounds. Further haematology and biochemistry revealed that he now had a raised white cell count $\left(18 \times 10^{9} / 1\right)$ with a leucocytosis, acute on chronic renal failure, and raised hepatic transaminases. His alanine transaminase was $171 \mathrm{U} / \mathrm{l}$ (range 7-33) and aspartate transaminase was $147 \mathrm{U} / \mathrm{l}$ (range 10-34). In addition

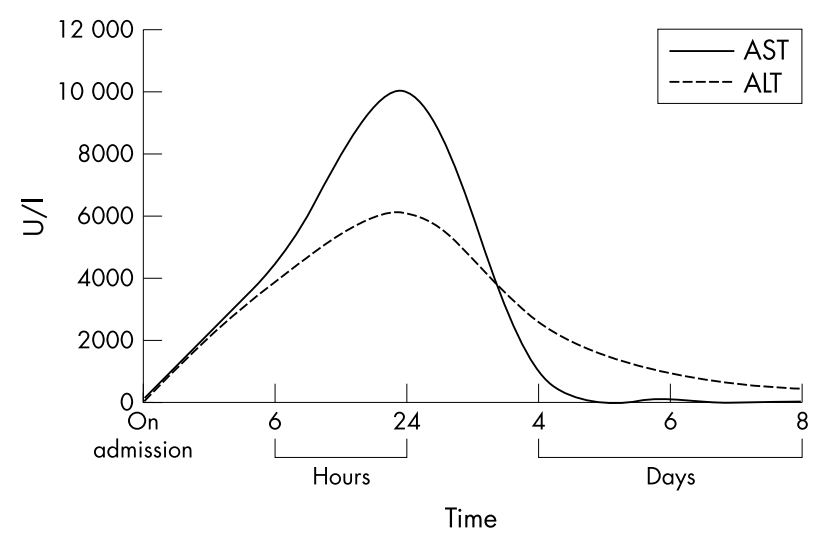

Figure 1 Trend of transaminases with time (ALT, alanine transaminase; AST, aspartate transaminase).

he had a raised amylase of $152 \mathrm{U} / \mathrm{l}$ (normal range 0-90 U/1), a metabolic acidosis ( $\mathrm{pH} 7.02$ ) with a raised serum lactate of 4.3 $\mathrm{mmol} / \mathrm{l}$ (range 0.6-2.4). His chest radiograph did not demonstrate gas under the diaphragm and abdominal radiography showed a non-specific gas pattern.

A provisional diagnosis of intestinal ischaemia was made, and was supported by the biochemical and haematological parameters. He was transferred to intensive care for further resuscitation and consideration for laparotomy. In view of his co-morbidity and likely diagnosis he was considered to be a high risk anaesthetic candidate and laparotomy was deferred. His prognosis appeared to be poor. Over the next three days his abdominal pain resolved completely but he was noted to have a dramatic deterioration in his liver function (fig 1). Further investigation included an abdominal ultrasound, which demonstrated free fluid over the liver and mucosal thickening of the large bowel in both flanks. An acute duodenal ulcer was found at gastroscopy. A non-invasive liver screen was normal. The diagnosis was now considered to be gastrointestinal haemorrhage due to an acute duodenal ulcer with hypotension causing secondary ischaemic hepatitis. His clinical recovery continued and within 14 days his liver function had normalised and he was discharged.

\section{DISCUSSION}

This is the first reported case of ischaemic hepatitis presenting with acute abdominal signs. Our patient demonstrates an atypical case of ischaemic hepatitis as the initial presentation was thought to be intestinal ischaemia. The diagnosis of intestinal ischaemia or infarction may be elusive. A high index of suspicion is necessary when assessing a patient with acute abdominal pain in the presence of arteriopathy or atrial fibrillation. ${ }^{7}$ Initially there may be a discrepancy between the symptoms, which may be severe, and the signs. Once intestinal infarction or perforation occurs abdominal signs become more apparent. The haematological and biochemical profile of patients with intestinal ischaemia is not specific or diagnostic. ${ }^{7}$ A leucocytosis, metabolic acidosis, raised serum 
lactate, hyperphosphataemia, and a raised amylase can all be

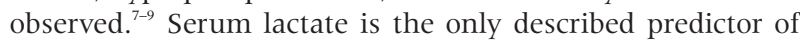
mortality. ${ }^{7}$ In a recent study of a 121 consecutive cases of intestinal infarction, of the patients who died (72/121), $81 \%$ had a serum lactate of greater than $5.2 \mathrm{mmol} / \mathrm{l}^{7}$ The mortality in reported case series range from $45 \%-100 \%{ }^{7-9}$

The biochemical and haematological abnormalities described in intestinal ischaemia may be identical to ischaemic hepatitis except in one aspect-markedly deranged transaminases do not occur. ${ }^{7-9}$ This is the key discriminating factor in the acute presentation. By recognising this, the clinician may avoid the necessity for laparotomy. The management of ischaemic hepatitis is supportive with correction of the underlying cause for hypotension.

It is possible that the patient in this case had both ischaemic hepatitis and intestinal ischaemia. However, this would seem unlikely given the high mortality described in intestinal ischaemia and the rapid improvement in the clinical status of our patient. In conclusion, we report the first case of ischaemic hepatitis presenting with acute abdominal signs. Recognition of markedly raised transaminases within this context may avoid unnecessary laparotomy.

\section{Authors' affiliations}

L Powell, S Tesfaye, D S Sanders, Department of Medicine, Royal Hallamshire Hospital, Sheffield

R Ackroyd, Department of Surgery

Correspondence to: Dr D S Sanders, Gastroenterology and Liver Unit, Room P14, P Floor, Royal Hallamshire Hospital, Sheffield S10 2JF, UK; d.s.sanders28@btopenworld.com

Submitted 16 January 2003

Accepted 24 February 2003

\section{Learning points}

- Ischaemic hepatitis is an uncommon condition precipitated by portal hypotension.

- Ischaemic hepatitis is recognised clinically by a marked deterioration in liver function and supportive management is indicated.

- Ischaemic hepatitis and intestinal ischaemia may have similar presenting features but the recognition of markedly raised transaminases can be the critical discriminating factor.

\section{REFERENCES}

1 Fushs S, Bogomolski-Yahalom V, Paltiel O, et al. Ischemic hepatitis: clinical and labatory observations of 34 patients. J Clin Gastroenterol 1998;26:183-6.

2 Gibson PR, Dudley FJ. Ischaemic hepatitis: clinical features, diagnosis and prognosis. Aust N Z J Med 1984;14:822-5.

3 Bynum TE, Boitnott JK, Maddrey WC. Ischemic hepatitis. Dig Dis Sci 1979:24:129-35.

4 Gitlin N, Serio KM. Ischemic hepatitis: widening horizons. Am J Gastroenterol 1992;87:831-6.

5 Rashed KA, McNabb WR, Lewis RR. Ischaemic hepatitis in the elderly. Gerontology 2002;48:245-9.

6 Henrion J, Descamps O, Luwaert $R$, et al. Hypoxic hepatitis in patients with cardiac failure: incidence in a coronary care unit and measurement of hepatic blood flow. J Hepatol 1994;21:696-703.

7 Newman TS, Magnuson TH, Ahrendt SA, et al. The changing face of mesenteric infarction. Am Surg 1998;64:611-6.

8 Ottinger LW, Austen WG. A study of 136 patients with mesenteric infarction. Surg Gynecol Obstet 1967;124:251.

9 Clark RA, Gallant TE. Acute mesenteric ischaemia: angiographic spectrum. Am J Roentgenol 1984;142:555-62. 